\title{
Hyperspectral imaging and multivariate analysis in the dried blood spots investigations
}

\author{
Alicja Majda $^{1} \cdot$ Renata Wietecha-Posłuszny ${ }^{1} \cdot$ Agata Mendys $^{2} \cdot$ Anna Wójtowicz $^{1} \cdot$ Barbara Łydżba-Kopczyńska ${ }^{2,3}$
}

Received: 9 January 2018 / Accepted: 12 March 2018 / Published online: 15 March 2018

(c) The Author(s) 2018

\begin{abstract}
The aim of this study was to apply a new methodology using the combination of the hyperspectral imaging and the dry blood spot (DBS) collecting. Application of the hyperspectral imaging is fast and non-destructive. DBS method offers the advantage also on the micro-invasive blood collecting and low volume of required sample. During experimental step, the reflected light was recorded by two hyperspectral systems. The collection of 776 spectral bands in the VIS-NIR range (400-1000 nm) and 256 spectral bands in the SWIR range $(970-2500 \mathrm{~nm}$ ) was applied. Pixel has the size of $8 \times 8$ and $30 \times 30 \mu \mathrm{m}$ for VIS-NIR and SWIR camera, respectively. The obtained data in the form of hyperspectral cubes were treated with chemometric methods, i.e., minimum noise fraction and principal component analysis. It has been shown that the application of these methods on this type of data, by analyzing the scatter plots, allows a rapid analysis of the homogeneity of DBS, and the selection of representative areas for further analysis. It also gives the possibility of tracking the dynamics of changes occurring in biological traces applied on the surface. For the analyzed 28 blood samples, described method allowed to distinguish those blood stains because of time of apply.
\end{abstract}

\section{Introduction}

As an excellent information carrier, blood is the most commonly analyzed biological material in diagnostic, toxicology, and forensic science. Typically, analyzed material is taken directly from the veins in a liquid form; however, in certain cases, it is more convenient to analyze blood stains created on a surface. Dried blood spot (DBS) sample collection technique offers a practical and simple alternative to traditional blood collection methods used to create human blood samples for analytes identification. The most benefits of using such a way of microsampling reduced sample amounts (eq. $10 \mu \mathrm{L}$ ). The next advantage is a low cost of biological sample storage and transport. Easy collecting by

Renata Wietecha-Posłuszny

wietecha@chemia.uj.edu.pl

1 Laboratory for Forensic Chemistry, Department of Analytical Chemistry, Faculty of Chemistry, Jagiellonian University, 2, Gronostajowa St., 30-387 Kraków, Poland

2 Laboratory of Analysis and Non-Destructive Investigation of Heritage Objects (LANBOZ), National Museum in Cracow, 1, 3-Maja St., 30-062 Kraków, Poland

3 Faculty of Chemistry, University of Wroclaw, 14, F. Juliot-Curie St., 50-383 Wrocław, Poland capillary or direct as a drop of blood, so specialists are not required in this case. Blood samples spotted on a DBS card can be stored for several months or years at room temperature if an appropriate humidity is maintained [1,2]. DBS testing is a form of biosampling, where blood samples are just blotted and dried on filter paper [3]. The described technique is used more and more frequently in the clinical diagnosis using variety of chromatographic methods $[4,5]$.

Blood stains analysis is as well an important issue in the field of forensic science. Methods used in the examination of biological traces at the crime scene are still developed and new techniques are introduced. Great potential for the surface analysis was recently recognized in the application of hyperspectral imaging (HSI) in a visible and near infra-red region of electromagnetic spectrum. Hyperspectral imaging is a reflection spectroscopy technique that gives information about reflectance spectrum for each point of the analyzed sample. Results are obtained and presented in the form of "hyperspectral cube", that is three dimensional data, with two spatial and one spectral dimension. It should be emphases that each pixel of the recorded image corresponds to one reflectance spectrum. Measurements are fast, non-invasive and can be performed in-situ, what gives the possibility to use this technique on material evidence without touching it, even directly at the crime scene. Application of HSI 
method in forensics was summarized e.g. by Edelman [6] and includes analysis of such traces as explosives [7], fingermarks, hair, drugs, inks, paints, pens [8], fibers, bruises [9] or blood stains [10]. It is used as well in analysis of documents [11] and works of art forgeries [12].

Absorption in the near infra-red region of electromagnetic spectrum is connected to transitions between vibrational molecular states, it contains information about organic molecules, such as proteins. Therefore, hyperspectral imaging in this spectral range gives possibility to distinguish between biological samples, even when they are not distinguishable by naked eye in visible region, due to the same color [10, 13]. Hyperspectral imaging combined with chemometric approach makes analysis easier by fast highlighting areas that contain the most interesting information [14]. Minimum noise fraction (MNF) is widely used in hyperspectral data analysis e.g. in the fields of remote sensing for geospatial applications [15], works of art investigations [16] or forensic science [9].

One of the important applications of hyperspectral imaging in blood stains analysis is the age estimation. For the blood stains, it is defined as the time elapsed from the moment of its creation. Estimation of the bleeding time may help crime scene investigators to determine the temporal aspects of a crime. There were many methods to study the age of the blood stains, such as oxygen electrode [17], RNA degradation [18], EPR [19] and HPLC [20]. In the HSI investigations, interpretation of the results is based on the differences in the spectra depending on the presence of hemoglobin derivatives [21].

The aim of this work was to apply the hyperspectral imaging methodology, as non-destructive and fast preliminary investigations of biological traces on the surface. HSI measurements supported by multivariate analysis methods are innovative tool used for determination of homogeneity, age, and desiccation of blood stains on DBS cards. By applying for the first time a hyperspectral imaging of properly prepared blood samples on DBS cards for the blood analysis, it is possible to estimate the drying time under standard conditions and blood stain area that can be punch to extraction. Presented methodology can be useful in analysis of other biological traces on the paper background e.g. in forensic investigations or in analysis of historical paper objects.

\section{Materials and methods}

\subsection{Sample characteristics}

Experiments were performed on blood stains created from $\mathrm{ARh}+$ blood samples received from a blood donation station. The ARh+ group was chosen as the most common in our population. The surface chosen for the deposition of the blood were DBS cards, type Whatman FTA Classic. Twentyeight samples consisting of $25 \mu \mathrm{L}$ of blood were pipetted singly onto DBS cards and then left to dry. Created spots are presented in Fig. 1. DBS cards were stored in the laboratory at a room temperature $\left(22{ }^{\circ} \mathrm{C}\right)$ and a relative humidity of $50 \%$. Table 1 presents the drying time for each single blood spot. Drying time was calculated until the moment of performing hyperspectral imaging measurement.

\subsection{Hyperspectral imaging}

In our experimental setup (Fig. 2), the investigated object was illuminated by a set of six halogen light sources placed at a $45^{\circ}$ angle relative to the normal, on each side of the camera. The reflected light was captured by two hyperspectral systems (SPECIM, Finland), working in a "push-broom" geometry and collecting 776 spectral bands in the VIS-NIR range (400-1000 $\mathrm{nm}$ ) and 256 spectral bands in the SWIR range (970-2500 nm). Pixel was the size of $8 \times 8$ and $30 \times 30 \mu \mathrm{m}$ for VIS-NIR and SWIR camera, respectively. For VIS-NIR detector, the exposure time was set to $70 \mathrm{~ms}$ and the signal was collected using a $10 \mathrm{~Hz}$ frame rate with a scanning speed of $0.6 \mathrm{~mm} / \mathrm{s}$. For SWIR detector, exposure time was set to $5 \mathrm{~ms}$, frame rate $-10 \mathrm{~Hz}$ and a scanning speed $-2.2 \mathrm{~mm} / \mathrm{s}$. Each recorded image was corrected for dark current (recorded with the camera shutter closed) and normalized using a white reference Spectralon bar (Labsphere, New Hampshire,

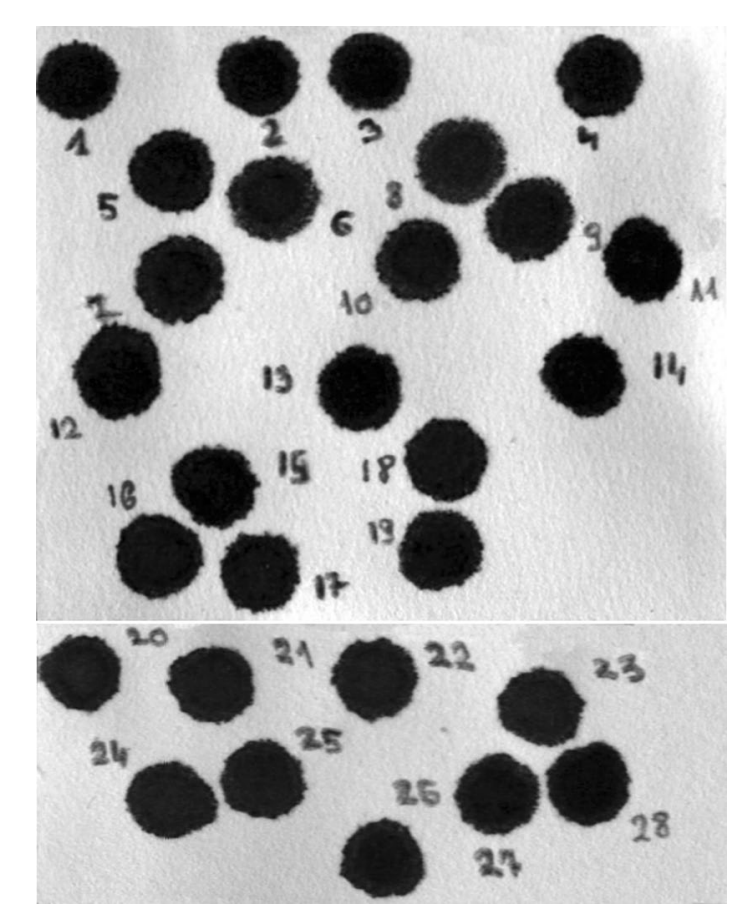

Fig. 1 Blood spots applied on DBS cards: a 1-19 samples, b 20-28 samples 
Table 1 Drying time for each single blood sample

\begin{tabular}{|c|c|c|c|}
\hline \multirow[t]{2}{*}{ Sample no. } & \multicolumn{3}{|c|}{ Age for a given measurement method } \\
\hline & VIS-NIR & & SWIR \\
\hline 1 & 29 D (days) & & \\
\hline 2 & $28 \mathrm{D}$ & & \\
\hline 3 & $27 \mathrm{D}$ & & \\
\hline 4 & $25 \mathrm{D}$ & & \\
\hline 5 & $22 \mathrm{D}$ & & \\
\hline 6 & $20 \mathrm{D}$ & & \\
\hline 7 & $18 \mathrm{D}$ & & \\
\hline 8 & $15 \mathrm{D}$ & & \\
\hline 9 & $13 \mathrm{D}$ & & \\
\hline 10 & $11 \mathrm{D}$ & & \\
\hline 11 & $8 \mathrm{D}$ & & \\
\hline 12 & $7 \mathrm{D}$ & & \\
\hline 13 & $6 \mathrm{D}$ & & \\
\hline 14 & $5 \mathrm{D}$ & & \\
\hline 15 & $4 \mathrm{D}$ & & \\
\hline 16 & $1 \mathrm{D}$ & & \\
\hline 17 & $18 \mathrm{H}$ (hours) & & \\
\hline 18 & $20 \mathrm{H}$ & & \\
\hline 19 & $0 \mathrm{M}$ (minutes) & & \\
\hline 20 & $290 \mathrm{M}$ & $275 \mathrm{M}$ & \\
\hline 21 & $225 \mathrm{M}$ & $210 \mathrm{M}$ & \\
\hline 22 & $165 \mathrm{M}$ & $150 \mathrm{M}$ & \\
\hline 23 & $105 \mathrm{M}$ & $90 \mathrm{M}$ & \\
\hline 24 & $75 \mathrm{M}$ & $60 \mathrm{M}$ & \\
\hline 25 & $45 \mathrm{M}$ & $30 \mathrm{M}$ & \\
\hline 26 & $30 \mathrm{M}$ & $15 \mathrm{M}$ & \\
\hline 27 & $15 \mathrm{M}$ & $1 \mathrm{M}$ & \\
\hline 28 & $0 \mathrm{M}$ & $-^{\mathrm{a}}$ & \\
\hline
\end{tabular}

${ }^{\text {a }}$ Sample measured exclusively by VIS-NIR

US). Both dark current and white reference frames were recorded with the abovementioned experimental parameters.

In this study, data analysis was conducted in two ways. First of all, the whole image was analyzed using tools implemented in Envi 5.0 (Exelis VIS, Colorado, US) software, namely Minimum Noise Fraction transform and $\mathrm{nD}$ visualizer. Subsequently, based on above-mentioned analysis, for each of the blood spots regions of interest (ROI) were defined, including at least 3000 pixels for VIS-NIR and 350 pixels for SWIR. Average reflectance spectra (R) extracted from ROI were converted to absorbance spectra using $A=\log (1 / R)$ relation. In the second step, the reflectance spectra of all the blood traces were analyzed by performing Principal Component Analysis using Statistica Data Miner software (StatSoft).

\subsection{Spectrum analysis}

Data obtained from hyperspectral imaging were stored in three dimensional file. Thanks to this, it was possible to get spectral characteristic from every pixel on the image. For a broader chemometric analysis, it was necessary to use an algorithm that transforms primary variables into new-mutually orthogonal. For this, we used PCA method, that allows reduction of the data dimensionality and graphic representation of the multidimensional dependence. Therefore, it is important to extract only those components that carry the most information about the data set. For these principal components, we can calculate its eigenvalue and total variance. The main components in this study were chosen making scree test-it consists to finding the place where the smooth decrease of eigenvalues appears to level off to the right of the plot. Significant components are located on the left of this point. Next steps of using PCA for hyperspectral data are to interpret and to define meaning of these components. Graphic interpretation of the dependence can be presented using scatter plot [22].

\subsection{Image analysis}

Prior to detail reflectance spectra analysis, full-image analysis was performed using minimum noise fraction (MNF) transform [19]. Unlike the PCA transform, the MNF orders principal components in terms of image quality, i.e., maximizes signal-to noise ratio. It consists of two PCA rotations and a noise whitening procedure. In a special case, when noise covariance matrix is identity matrix, MNF transform reduces to PCA. After performing MNF transformation, the most noisy bands can be removed or smoothed. This method allows to reduce the dimensionality of the data and defines new uncorrelated principal components.

New uncorrelated bands, obtained as a result of forward MNF transform, can be visualized in a form of gray-scale images or, by choosing three bands corresponding to three principal components, false-RGB images can be created. ENVI software gives another possibility to graphically analyze MNF results, using nD-visualizer tool to create 3D scatter plot in a space defined by three chosen principal components. To pre-compare traces of blood without the chemical interpretation of spectra, MNF algorithm was used directly on the hyperspectral cube. Analyzed region was limited only to blood stains, without showing numbers written with ink traces, by applying masks on the images. 


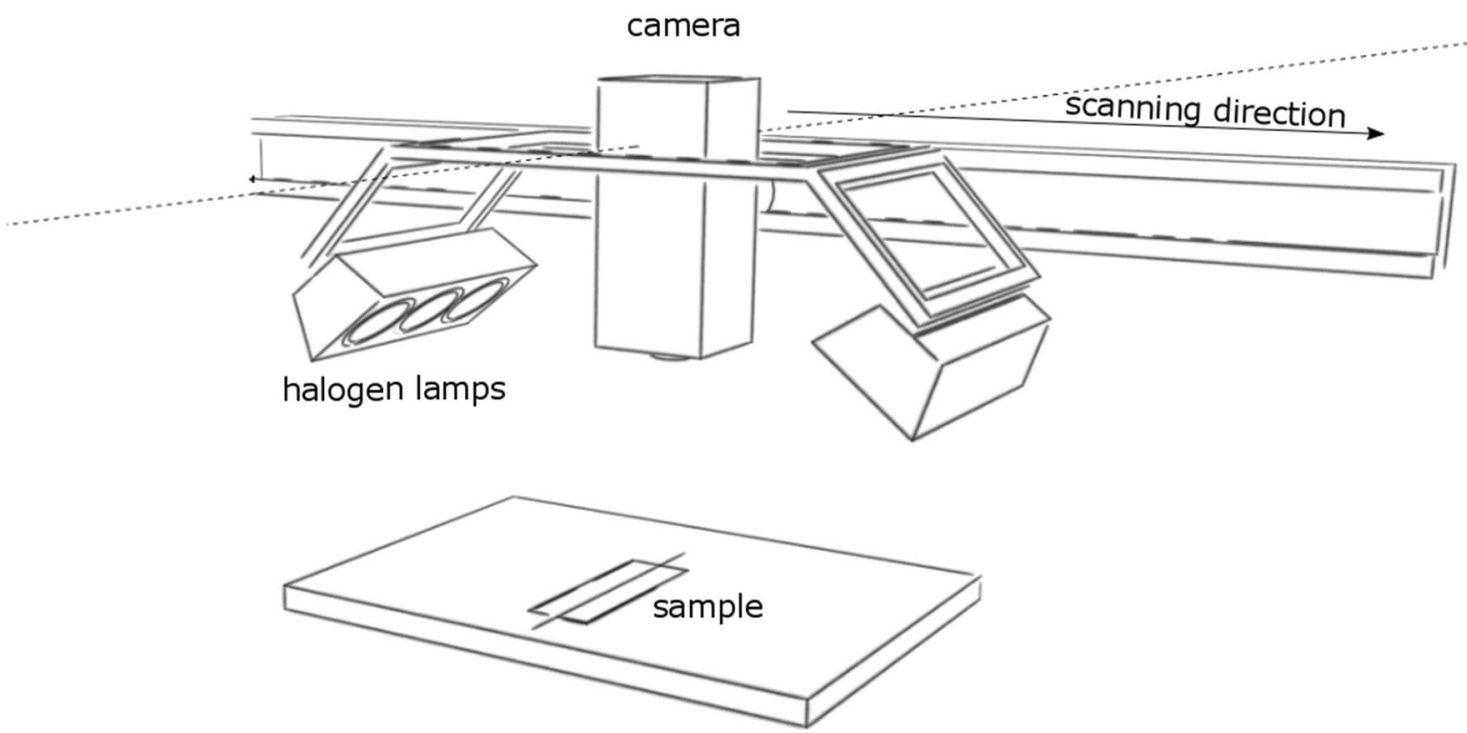

Fig. 2 Hyperspectral imaging setup

\section{Results and discussion}

\subsection{VIS-NIR image analysis}

Principal components obtained from the MNF transform of images recorded in VIS-NIR range were analyzed. First principal component distinguishes only area of paper background, and does not contain any information about blood stains. Based on second, third, and fourth principal component-false RGB images were created, as presented in Fig. 3. Fast visual analysis of the images allows to classify blood stains into main groups: five groups for samples 1-19 (samples nos. 1-5; 6-10; 11-15; 16-17, and 18-19) and five groups for samples 20-28 (samples nos. 20-23; 26-27; unclassified 24; 25 and 28). Figure 4 shows the assignment to groups according to the blood stain age.

The analysis resulted in isolation of characteristic areas 'coffee rings' on the outskirts of blood stains. In addition, large homogeneous areas located in the center of stains can be noticed. The obtained results can be used in studies of blood traces on surface, which requires a representative sample. Moreover, in the case of analysis of biological material deposited on DBS cards, it is possible to estimate surface to punch and extraction to quantitative analysis.

Further analysis steps were aimed at finding the correlation between classification into groups and information about chemical composition, carried by the spectra collected from the blood stains.

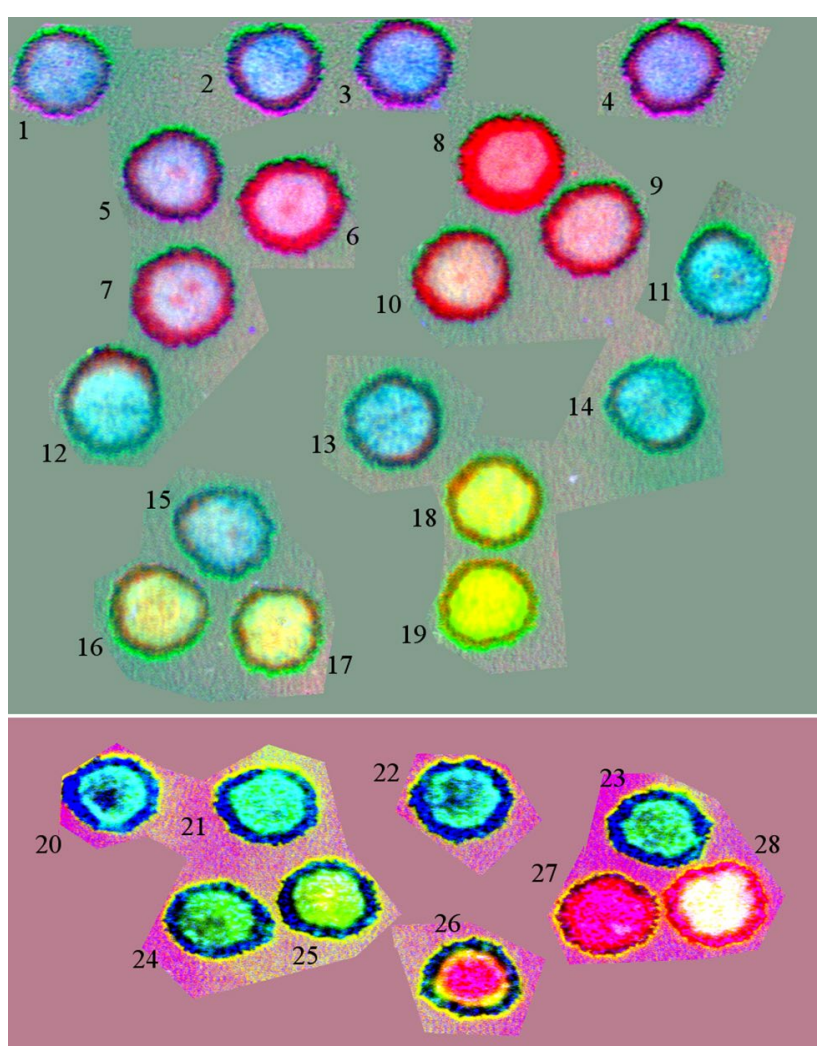

Fig. 3 Blood spot samples after minimum noise fraction algorithm in RGB based on a PC2-PC3-PC4, b PC2-PC3-PC5 


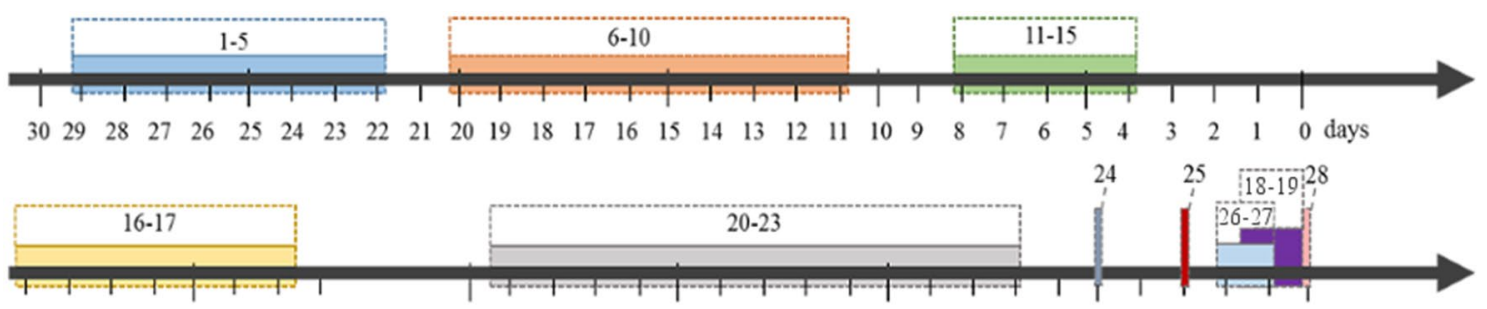

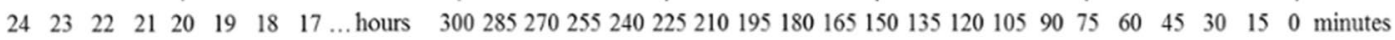

Fig. 4 Assignment to groups, according to the interval time of applied blood onto DBS card

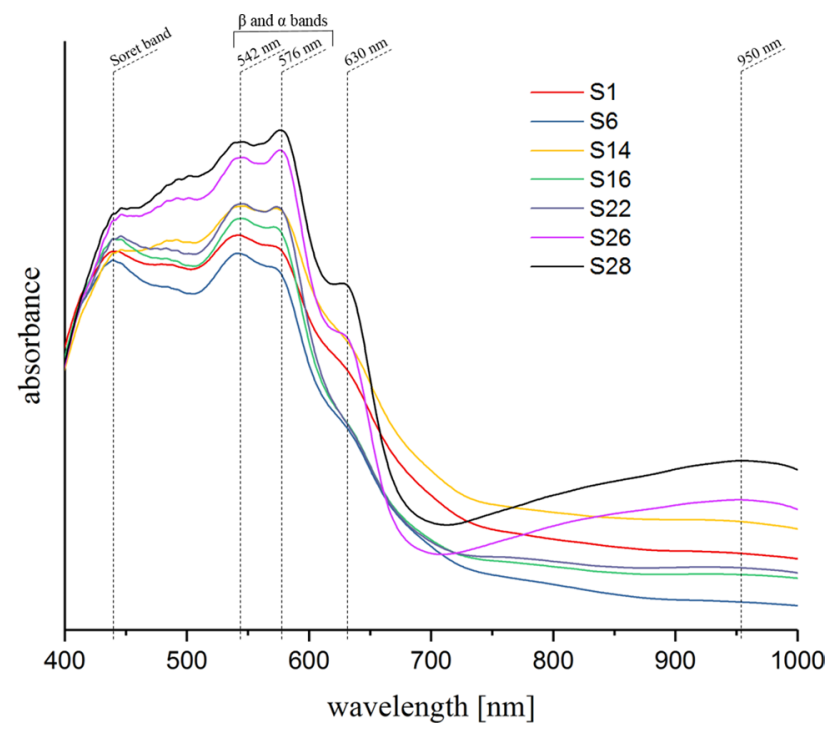

Fig. 5 Average spectrum for each blood spot in Vis-NIR range

\subsection{VIS-NIR spectra analysis}

Due to the changes in composition of blood stains with time, differences arising from the presence of hemoglobin derivative such as oxy-hemoglobin, met-hemoglobin, hemichrome and water should be observed. Fresh blood contains mainly hemoglobin saturated with oxygen $-\mathrm{HbO}_{2}$. The visible absorption spectrum of $\mathrm{HbO}_{2}$ has one strong peak at $\sim 414 \mathrm{~nm}$ called the Soret band and two weaker peaks at $\sim 542$ and $576 \mathrm{~nm}$ referred to as $\beta$ and $\alpha$ bands, respectively. With the increasing age of blood stain, $\mathrm{HbO}_{2}$ oxidizes to methemoglobin (met-Hb). Subsequently, due to decreasing availability of cytochrome b5 needed for reduction of met$\mathrm{Hb}$, met-Hb denaturates to hemichrome (HC) [23]. These derivatives have different absorption properties and cause changes in the spectrum mainly in the region of the $\alpha$ and $\beta$ bands. With time, one can observe decreasing intensity of $\alpha$ and $\beta$ bands as well as appearance of peak $630 \mathrm{~nm}-$ corresponding to met-Hb absorption [24].

The spectral characteristics of the blood spots in VIS-NIR range are shown in Fig. 5. Each plot represents the absorption spectrum averaged over previously selected ROIs, from the homogeneous central area of the blood spots. For clarity, only one spectrum from each of distinguished groups is shown. The blood spectra that are different from other ones, can be selected visually from the whole samples were S26 and S28. Those samples are younger than $30 \mathrm{~min}$, so the main difference is probably due to the presence of water and oxyhemoglobin. In addition, changes in intensity of $\beta$ and $\alpha$ bands are very clear for all spectra. It depends on the concentration of each hemoglobin derivatives and thus the age of the blood samples.

It should be noticed that visual assessment of the spectrum is very subjective and does not allow to obtain detailed distinguish. To observe the changes in whole spectral range not only selected area, chemometric analysis should be used.

\subsection{Chemometric application}

In the following steps, PCA based on average spectra defined by ROIs was used to detect a correlation between the analyzed blood stains and specific spectrum changes, dependent on the time of blood application on the DBS card. The first three principal components extracted by the scree plot explain more than $97 \%$ of all variation in examined dataset (PC1 71.08\%; PC2 20.54\%; PC3 6.20\%; PC4 1.40\%; PC5 $0.41 \%$; PC6 $0.26 \%$; PC7 0.05\%). It is worth noticing that the first principal component carries information about differentiation between samples and background. Graphical representations of samples correlation were presented as scatter plots and explained according to the procedure used in the literature [8]. Scatter plots were prepared using first and second (Fig. 6a), second and third (Fig. 6b) principal components plotted together. Samples on the figures were marked by colors resulting from the previous MNF grouping. Analysis of those graphs shows that the grouping resulting from the PCA method for ROIs largely confirms earlier used MNF analysis for whole image. The greatest differences are noticeable between fresh samples-S25, S26, S27, and S28, thus younger than $45 \mathrm{~min}$. However, it can be observed that samples S1-S4, S11-S15 and S5-S10, S16-S17, and S18-S24 have similar correlations with 

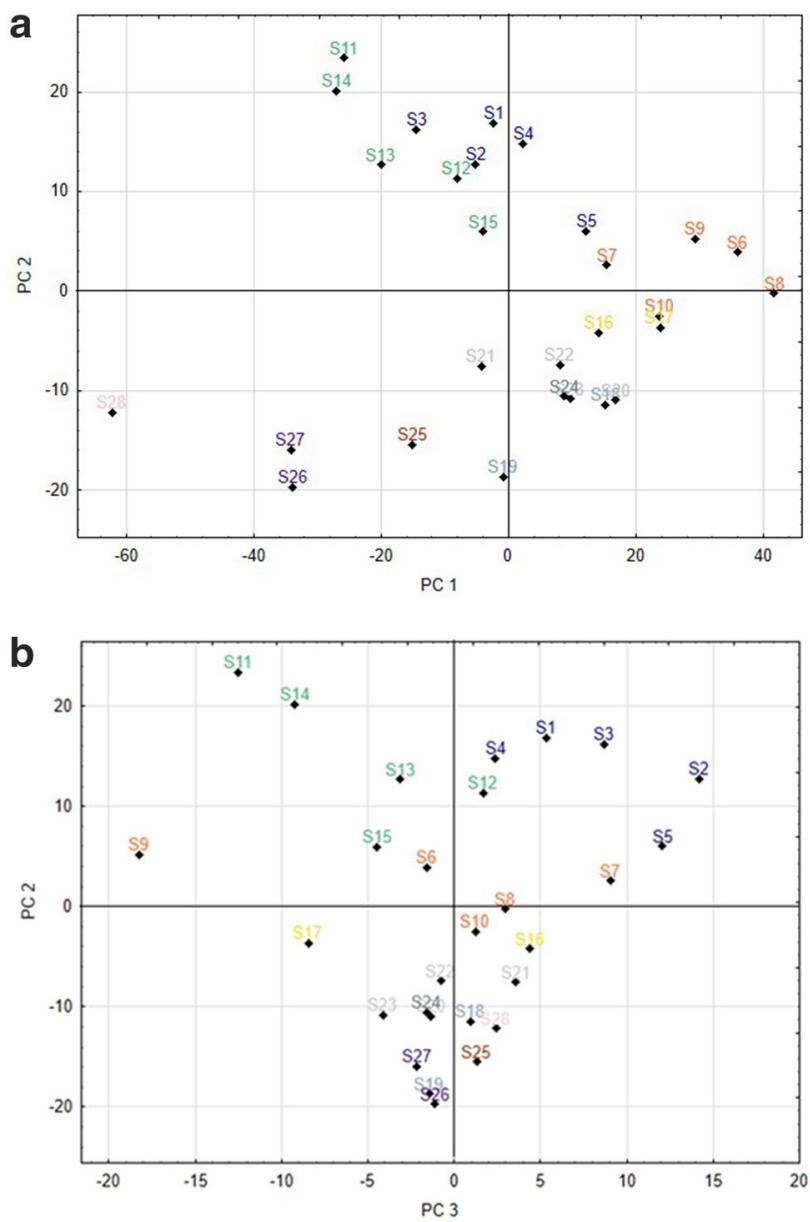

Fig. 6 a Scatter plot for PC 1 and PC 2. b Scatter plot for PC 2 and PC 3

the principal components; therefore, their differentiation between groups and within the groups is difficult using only this method. Based on those results of the analysis carried out in the Vis-NIR range, further study in less efficiently absorbed NIR light (720-2500 nm) has been made using only MNF method.

\subsection{SWIR image analysis}

The spectral characteristics of the chosen blood spots ( $\mathrm{S} 1$, S6, S14, S16, S22, S26, and S27) in SWIR range are shown in Fig. 7. This plot, and previous analysis showed that the biggest changes occur in the first hours after application of the sample blood on the surface. Therefore, further studies on changes occurring in the samples 20-27 were performed by analyzing hyperspectral image in the SWIR spectral range. The age of this samples were presented in Table 1. MNF analysis was performed on the hyperspectral cube, with masked areas containing the ink labels. Based on principal components obtained in the MNF transform, 3D

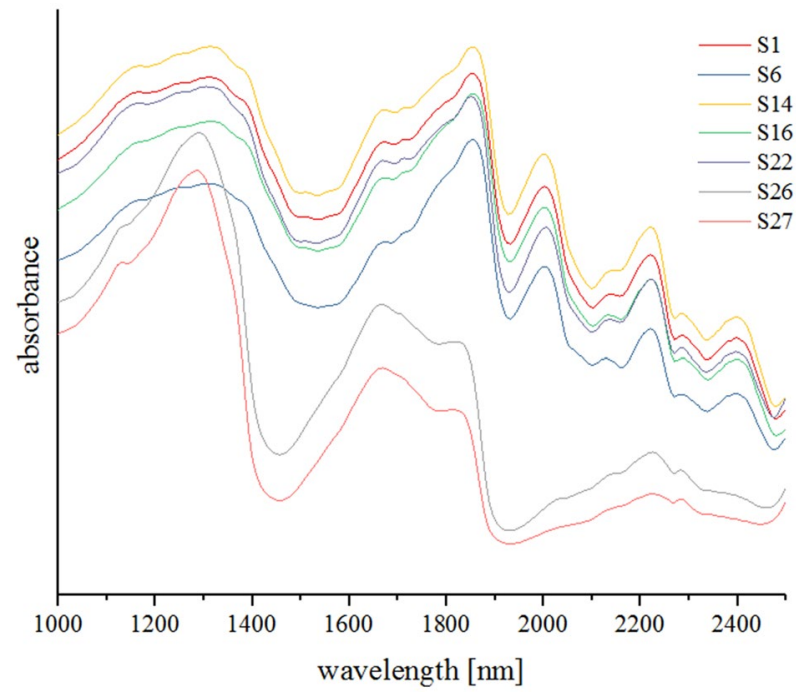

Fig. 7 Average spectrum for each blood spot in SWIR range

scatter plot was created (using $\mathrm{nD}$ visualizer tool from ENVI software). As can be seen in Fig. 8, the scatter plot in 3d space created by PC1, PC2, and PC3 has a shape of five long curved 'arms', connected with each other at one end. Each point corresponds to one pixel at the hyperspectral image. Distance between points corresponds to spectral distance between spectra, i.e., the shorter the distance between points, the more similar spectra. Thus, points are grouped according to their spectral similarity.

Groups of points were marked on the edges of the distribution, and then mapped on the original image, using

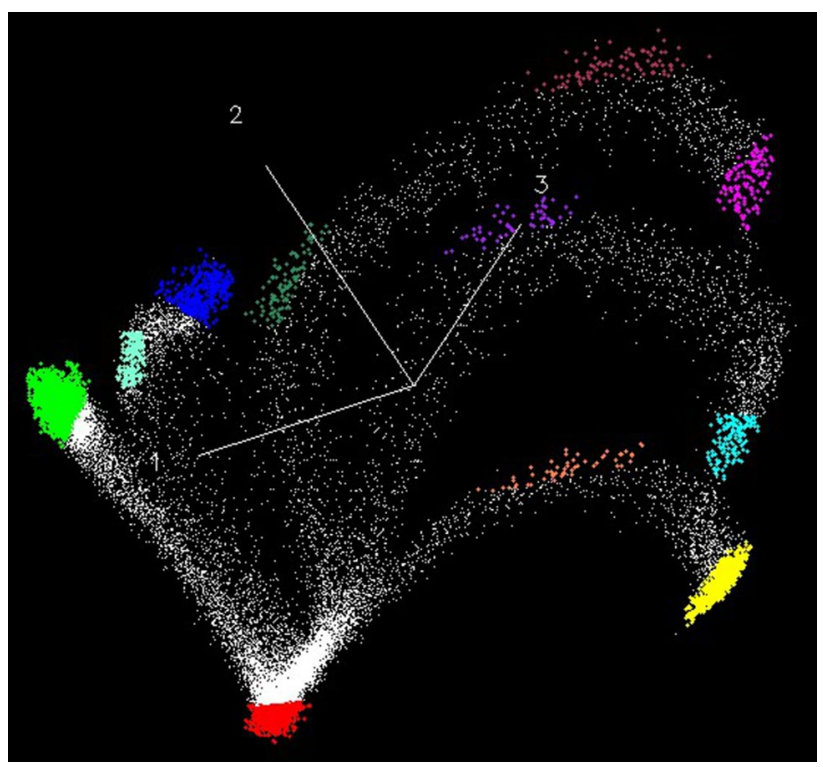

Fig. 8 3D visualization for samples $20-28$ in Vis-NIR spectrum range 


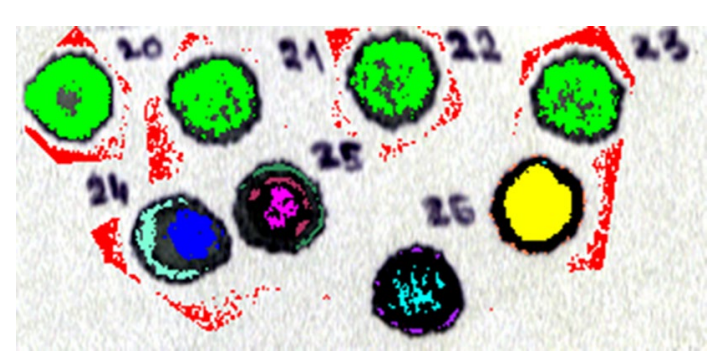

Fig. 9 Mapping of groups of pixels marked on 3D visualization for samples 20-28

respective colors (Fig. 9). The results confirm the dynamic changes occurring in the first hours after extravasation. Large spectral distance between samples no. 24-27 is clearly observed, while samples $20-23$ are found at the same area of the scatter plot (bright green points). It follows that, for further analysis requires cutting part of sample, under similar conditions, $90 \mathrm{~min}$ of drying is sufficient (the time given for sample S23 according to data from Table 1 for SWIR range measurement). All 'arms' are connected by the points corresponding to the spectra of background paper (red points).

Furthermore, points from one of the scatterplots 'arms' (corresponding to spot no. 25) were grouped (Fig. 10), to follow the correlation between scatter plot shape and spatial distribution of points on the blood traces (Fig. 11). The observed changes are due to the gradual drying of blood spots and differences in the contents of hemoglobin derivatives-mainly oxy- and met-hemoglobin.

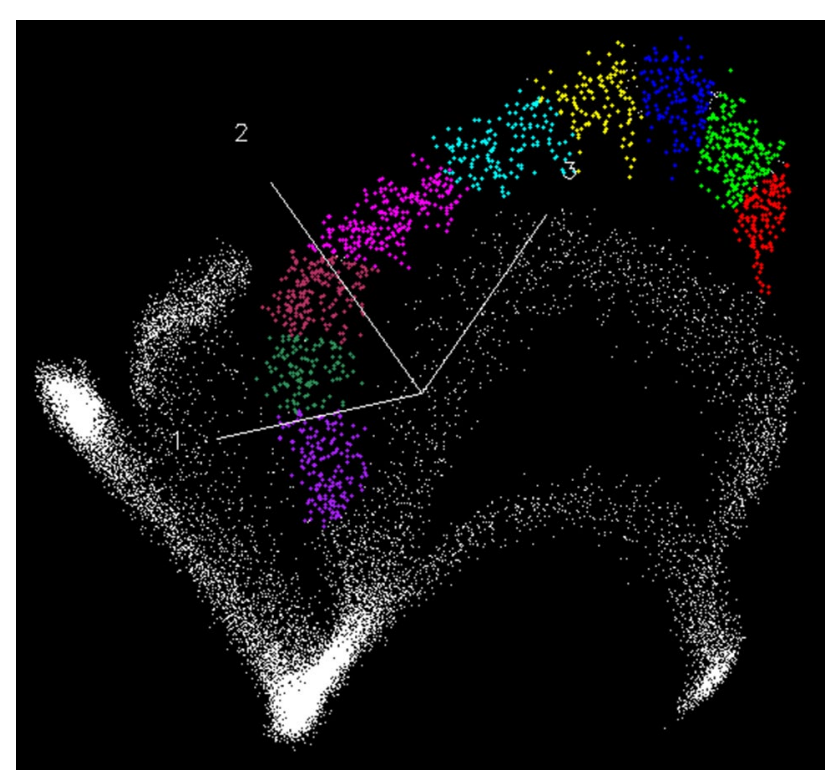

Fig. $103 \mathrm{D}$ visualization for sample no. 25 in Vis-NIR spectrum range

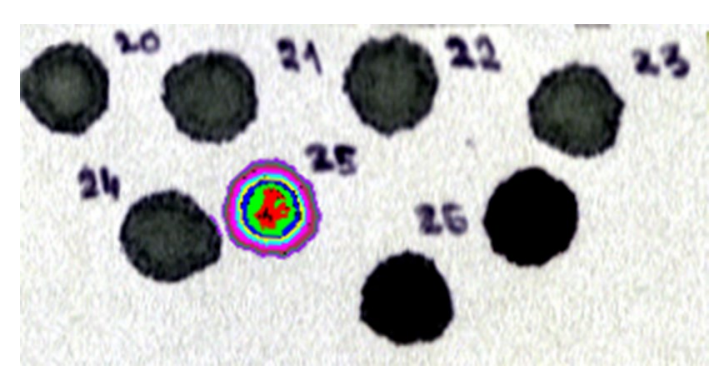

Fig. 11 Mapping of groups of pixels marked on 3D visualization for sample no. 25

\section{Conclusion}

Method applied in this study is non-destructive, effective, and fast. MNF algorithm successfully distinguished blood spots according to the age in time interval between 0 and 29 days and shorter between minutes range. It provides information on the dynamics of the processes occurring during drying of the blood spots. The hyperspectral imaging methodology coupled with proposed way of investigation could be used especially such as preliminary tests of homogeneity of DBS and to choose places to punch to analysis using for example separation method such as: LC or CE. Moreover, in the future, the above-mentioned methodology will be developed-to be used for analytes identification in the DBS samples. Due to non-invasive character, our proposed methodology of age estimation of the blood stains can be used in forensic research on the crime scene.

Open Access This article is distributed under the terms of the Creative Commons Attribution 4.0 International License (http://creativeco mmons.org/licenses/by/4.0/), which permits unrestricted use, distribution, and reproduction in any medium, provided you give appropriate credit to the original author(s) and the source, provide a link to the Creative Commons license, and indicate if changes were made.

\section{References}

1. R.J. Meesters, G.P. Hooff, State-of-the-art dried blood spot analysis: an overview of recent advances and future trends. Bioanalysis 5, 2187-2208 (2013). https://doi.org/10.4155/bio.13.175

2. M.L. Qi, P. Wang, Y.X. Leng, J.L. Gu, R.N. Fu, Erratum: simple HPLC method for simultaneous determination of acetaminophen, caffeine and chlorpheniramine maleate in tablet formulations (Chromatographia (2002) 56 (295-298)). Chromatographia 57, 122 (2003) https://doi.org/10.1007/BF02497491

3. M.S. Lee, W. Li, Dried Blood Spots: Applications and Techniques. Wiley Oxford (2014)

4. M.V. Antunes, M.F. Charão, R. Linden, Dried blood spots analysis with mass spectrometry: potentials and pitfalls in therapeutic drug monitoring. Clin. Biochem. 49, 1035-1046 (2016). https://doi. org/10.1016/j.clinbiochem.2016.05.004 
5. A.T. Salimo, J. Ledwaba, A. Coovadia, E.J. Abrams, K.-G. Technau, L. Kuhn, L. Morris, G.M. Hunt, The use of dried blood spot specimens for HIV-1 drug resistance genotyping in young children initiating antiretroviral therapy. J. Virol. Methods 223, 30-32 (2015). https://doi.org/10.1016/j.jviromet.2015.07.005

6. G.J. Edelman, E. Gaston, T.G. van Leeuwen, P.J. Cullen, M.C.G. Aalders, Hyperspectral imaging for non-contact analysis of forensic traces. Forensic Sci. Int. 223, 28-39 (2012). https://doi. org/10.1016/j.forsciint.2012.09.012

7. M. Fernández, J.M. de la Ossa, C. Amigo, García-Ruiz, Detection of residues from explosive manipulation by near infrared hyperspectral imaging: a promising forensic tool. Forensic Sci. Int. 242, 228-235 (2014). https://doi.org/10.1016/j.forsciint.2014.06.023

8. D.K. Chlebda, A. Majda, T. Łojewski, J. Łojewska, Hyperspectral imaging coupled with chemometric analysis for non-invasive differentiation of black pens. Appl. Phys. A. 122, 957 (2016). https ://doi.org/10.1007/s00339-016-0494-9

9. L.L. Randeberg, E.L.P. Larsen, L.O. Svaasand, Characterization of vascular structures and skin bruises using hyperspectral imaging, image analysis and diffusion theory. J. Biophoton. 3, 53-65 (2010). https://doi.org/10.1002/jbio.200910059

10. R.L. Schuler, P.E. Kish, C.A. Plese, Preliminary observations on the ability of hyperspectral imaging to provide detection and visualization of bloodstain patterns on black fabrics. J. Forensic Sci. 57, 1562-1569 (2012). https://doi.org/10.111 1/j.1556-4029.2012.02171.x

11. C.S. Silva, M.F. Pimentel, R.S. Honorato, C. Pasquini, J.M. PratsMontalbán, A. Ferrer, Near infrared hyperspectral imaging for forensic analysis of document forgery. Analyst 139, 5176-5184 (2014). https://doi.org/10.1039/c4an00961d

12. A. Polak, T. Kelman, P. Murray, S. Marshall, D.J.M. Stothard, N. Eastaugh, F. Eastaugh, Hyperspectral imaging combined with data classification techniques as an aid for artwork authentication. J. Cult. Herit. 26, 1-11 (2017). https://doi.org/10.1016/j. culher.2017.01.013

13. G. Edelman, V. Manti, S.M. Van Ruth, T. Van Leeuwen, M. Aalders, Identification and age estimation of blood stains on colored backgrounds by near infrared spectroscopy. Forensic Sci. Int. 220, 239-244 (2012). https://doi.org/10.1016/j.forsciint.2012.03.009

14. J. Yang, D.W. Messinger, J.J. Mathew, R.R. Dube, Comparison of algorithms for blood stain detection applied to forensic hyperspectral imagery. SPIE Proc. Algorithms Technol. Multispectral, Hyperspectral Ultraspectral Imag. XXII. 9840, 98400X (2016). https://doi.org/10.1117/12.2223293

15. F. Frassy, G. Dalla Via, P. Maianti, A. Marchesi, F. Rota Nodari, M. Gianinetto, Minimum noise fraction transform for improving the classification of airborne hyperspectral data: two case studies. Proc. 5th Work. Hyperspectral Image Signal Process. Evol. Remote Sens. (WHISPERS), 2013, pp. 1-4 (2013)

16. J.K. Delaney, P. Ricciardi, L.D. Glinsman, M. Facini, M. Thoury, M. Palmer, E.R. de la Rie, Use of imaging spectroscopy, fiber optic reflectance spectroscopy, and X-ray fluorescence to map and identify pigments in illuminated manuscripts. Stud. Conserv. 59, 91-101 (2014). https://doi.org/10.1179/2047058412Y.00000 00078

17. T. Matsuoka, T. Taguchi, J. Okuda, Estimation of bloodstain age by rapid determinations of oxyhemoglobin by use of oxygen electrode and total hemoglobin. Biol. Pharm. Bull. 18 1031-1035. http://www.ncbi.nlm.nih.gov/pubmed/8535389. Accessed 17 Aug 2017 (1995)

18. M. Bauer, I. Gramlich, S. Polzin, D. Patzelt, Quantification of mRNA degradation as possible indicator of postmortem interval—a pilot study., Leg, Med. (Tokyo), vol 5, pp. 220-227. http:// www.ncbi.nlm.nih.gov/pubmed/14602165. Accessed 17 Aug $2017(2003)$

19. Y. Fujita, K. Tsuchiya, S. Abe, Y. Takiguchi, S. Kubo, H. Sakurai, Estimation of the age of human bloodstains by electron paramagnetic resonance spectroscopy: long-term controlled experiment on the effects of environmental factors. Forensic Sci. Int. 152, 39-43 (2005). https://doi.org/10.1016/j.forsciint.2005.02.029

20. R. Kumagai, Analysis of hemoglobin in bloodstains using highperformance liquid chromatography. Nihon Hoigaku Zasshi. 47 213-219. http://www.ncbi.nlm.nih.gov/pubmed/8345684. Accessed 17 Aug 2017 (1993)

21. R.H. Bremmer, K.G. De Bruin, M.J.C. Van Gemert, T.G. Van Leeuwen, M.C.G. Aalders, Forensic quest for age determination of bloodstains. Forensic Sci. Int. 216, 1-11 (2012). https://doi. org/10.1016/j.forsciint.2011.07.027

22. Big Data, Data Mining, Predictive Analytics, Statistics, StatSoft Electronic Textbook (2017). http://www.statsoft.com/Textbook. Accessed 17 Aug 2017

23. W. Zijlstra, A. Buursma, W. Meeuwsen-van der, Roest, Absorption spectra of human fetal and adult oxyhemoglobin, de-oxyhemoglobin, and methemoglobin. Clin. Chem. 37, 1633-1638 (1991). https://doi.org/10.1109/CVPR.1999.784637

24. F. Shihana, D.M. Dissanayake, N.A. Buckley, A.H. Dawson, A simple quantitative bedside test to determine methemoglobin. Ann. Emerg. Med. 55, 184-189 (2010). https://doi.org/10.1016/j. annemergmed.2009.07.022 